\title{
Institutional Merit Award Fundacion Valle de Lili, Cali, Colombia
}

Panamerican Journal of Trauma, Critical Care \& Emergency Surgery (2020): 10.5005/jp-journals-10030-1270

The Merit Award from Fundacion Valle del Lili is granted to the physician of the year, who stands for his commitment to the institution, human quality, leadership, contribution to development, service vocation, responsibility, and personal relationships, and who complies with all the institutional values; within these, there is an important one, which is the integrity that leads to the ethics as a fundamental value to all our collaborators. Additionally, this physician gives even more, he creates, innovates, and this leads the institution to be at a high level in our country and in Latin America.

This distinction is awarded to Doctor Carlos Alberto Ordoñez D.

December 18, 2019, Cali, Colombia.

Dr Marcela Granados,

Medical Director

Fundacion Valle de Lili, Cali, Colombia.

\section{Doctor Carlos Ordoñez's Speech}

I want to thank Mayor Maurice Armitage for all he's done for the city of Cali and for his presence at this award ceremony. I feel honored receiving this distinction and grateful to Fundacion Valle del Lili and its administrative staff: Dr Borrero, Dr Marcela Granados, Dr Quintero, and Dr Watemberg, for having shared these past 25 years with me. I want to thank my family, my parents, my wife Sandra, and my children Sebastian, Alejandro, Paulina, and Jacobo, and all my friends and coworkers.

I want to dedicate a few words to the new upcoming generations:

Dr Adams Cowley, Founder of the Shock Trauma Center, stated that "if I can get to you, stop the bleed and restore the blood pressure in the first hour after the accident, I may be able to save you." This ended up being known as the "Golden Hour." Dr Cowley was never satisfied and during the inauguration of his new trauma center he said, "We have done a great work, but we haven't done enough. People are still dying unnecessarily."

These words were said more than 40 years ago and I had the fortune of meeting him and these words left a huge impression on me. Today, despite all the work done by surgeons all over the world, including our own, people are still dying unnecessarily. But, how can we lower this burden further?

Einstein said, "Madness is to do the same thing over and over again expecting different results," but this is exactly the pattern that we fall into repeatedly. We dare not do something different from what is considered the "right way" because we have been taught no differently, it's what the literature says, its dogma or the established paradigm, and to dare to do something different is hard, difficult and at times exhausting. The potential criticism, opposition, lawsuits, audits, and the disdain of our fellow colleagues refrain us from such actions.

We are taught to live in fear, and this inhibits change. What is needed is that we need to dream the impossible. To accomplish this, it is necessary to fight, to persevere, to risk, not to have fear of failure, to be resilient, and to adapt to adverse situations. What distinguishes leaders from followers is their creativity and innovation.

We must have firm and clear convictions. In trauma, the patient's life is in your hands; if the patient gets to you alive, ¡He must stay alive! So you have to have the knowledge, the skills, and the confidence to overcome the odds.

Cali has been a violent city for over 40 years. In the 1990s, it was one of the most violent cities of the world, with a homicide rate higher than 125 per 100,000 inhabitants. For us to understand the magnitude of this problem, we must remember that rates higher than 20 per 100,000 inhabitants are considered an epidemic. Nowadays the rates have lowered but remain around the 50 per 100,000 habitants range.

iiBut we have turned this misfortune to fortune!! Because opportunities are always hidden among difficulties. In the 1980s, when we created our trauma team, the mortality of our severely injured patients with gunshot wounds averaged around 70-80\%, but nowadays only $20 \%$ of these patients die at our institution.

Nelson Mandela once said that education is the most powerful weapon to change the world. We have applied this mantra in trauma, acute care surgery, and critical care. We have realized that it is not enough to achieve survival for each one of our critically injured trauma patients, but also to transform this experience in science for others around the globe to learn from our vast experience and the innovations we dare to achieve. Through our publications, we have contributed to the education of other surgeons around the world with the ultimate goal of saving lives beyond our city limits and reaching the farthest corners of the globe. We have to be surgeons who leave a mark and not just scars.

() The Author(s). 2020 Open Access This article is distributed under the terms of the Creative Commons Attribution 4.0 International License (https://creativecommons. org/licenses/by-nc/4.0/), which permits unrestricted use, distribution, and non-commercial reproduction in any medium, provided you give appropriate credit to the original author(s) and the source, provide a link to the Creative Commons license, and indicate if changes were made. The Creative Commons Public Domain Dedication waiver (http://creativecommons.org/publicdomain/zero/1.0/) applies to the data made available in this article, unless otherwise stated. 
A leader must form future leaders; the new generation of surgeons must keep innovating and breaking paradigms until we are able to reduce mortality at minimum. We have created a brotherhood of trauma surgeons that expand beyond the borders of Colombia and Latin America.

How do you build a true academic career? First of all, you have to truly enjoy and love what you do. Your experience and knowledge must be at the reach of everyone; it must become a scientific paper. This legacy of knowledge is essential for the advancement and care of our fellow men.

Dr Alonso Gomez, emeritus professor of intensive care, one day told me that the most important thing in life is to be able to create a school and that is exactly what we are building here, a school of Colombian Trauma and Acute Care Surgeons with a solid ground of knowledge in intensive care who are committed to their patients, to their institution, and to their country (Figs 1 and 2).

Currently, the wars of Iraq and Afghanistan have taught us about the first 10-15 platinum minutes of hemorrhage control, which is essential to improve the outcomes. Surgeons from all over the world are working hard to achieve this goal. Let's show the world that we are capable and willing to achieve these goals!

Einstein said, "The world is a dangerous place to live, not because of the people who are evil, but because of the people who don't do anything about it."

Welcome to the future.

Thank you very much.

Carlos Alberto Ordoñez D, MD, FACS

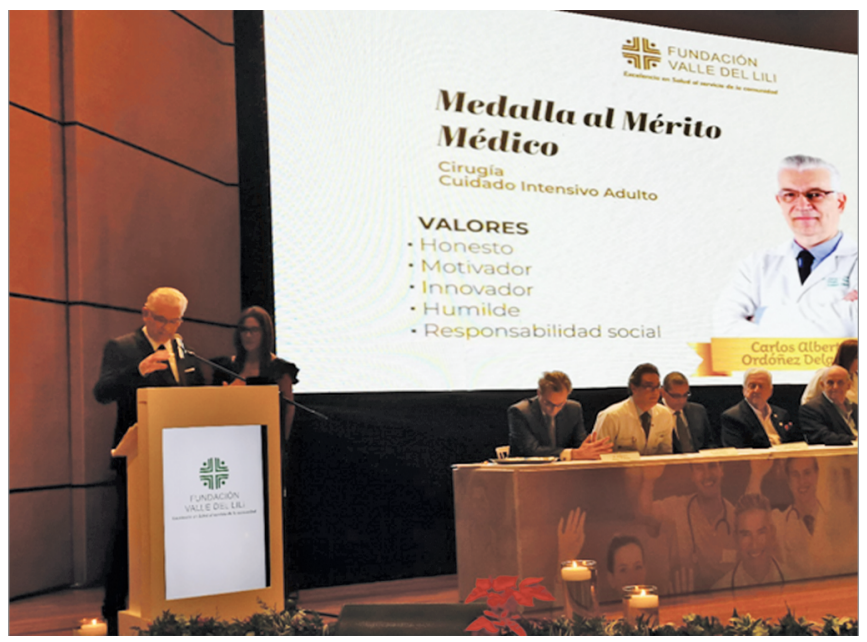

Fig. 1: Institutional Merit Award ceremony, in the screen are displayed the values that characterize the awarded: honesty, motivation, innovation, humility and social responsibility. On the left, Dr Carlos A Ordoñez

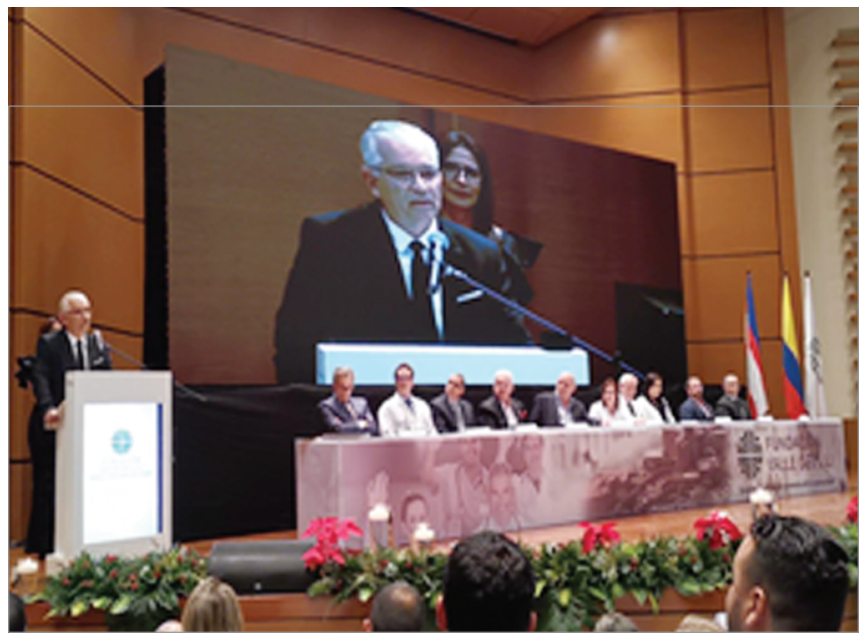

Fig. 2: Dr Carlos A Ordoñez speech during the ceremony. In the panelist table are found the directive staff and the mayor Maurice Armitage 


\section{Medalla Al Mérito Institucional Fundación Valle del Lili, Cali, Colombia}

La Medalla al Mérito Institucional en la Fundación Valle del Lili se otorga al médico del año, quien se haya destacado por la excelencia en compromiso con la institución, calidad humana, liderazgo, contribución al desarrollo, vocación de servicio, responsabilidad y relaciones interpersonales. Que además cumpla a cabalidad con todos los valores de la Fundación, dentro de los cuales hay uno muy importante que es la integridad como persona que nos lleva a que la ética sea un valor fundamental en todos nuestros colaboradores. Adicionalmente al estar alineado con la estrategia de la Fundación, entregue mucho más, entregue innovación, entregue creación, en procedimientos y en procesos, que lleven a la Fundación a estar al nivel nacional y latinoamericano en la que hoy nos encontramos.

Se concede la distinción al Doctor Carlos Alberto Ordoñez D.

Diciembre 18 de 2019, Cali.

Dra. Marcela Granados

Directora Médica y Académica

Fundación Valle de Lili, Cali, Colombia.

\section{Discurso Doctor Carlos A Ordoñez}

Señor alcalde Maurice Armitage, quiero darle un saludo y agradecerle por todo lo que ha hecho por Cali en estos años. Me siento muy emocionado de recibir este reconocimiento, esta medalla al merito, muy agradecido, con la Fundación y todos sus directivos, gracias Dr Borrero, Dra. Marcela Granados, Dr Quintero, Dr Martin Watemberg y a todos por haber tenido la paciencia y haber compartido estos 25 años de trabajo. Gracias a mi familia, a mis padres, a mi esposa Sandrita, y a mis hijos Sebastián, Alejandro, Paulina y Jacobo, y a todos mis amigos y compañeros.

Quiero en estos minutos decir unas palabras, sobre todo para la generación que viene en camino.

El Dr Adams Cowley, fundador del Shock Trauma Center, en Baltimore, Maryland, dijo "Si puedo llegar a ti, detener el sangrado y restaurar la presión arterial en la primera hora después del accidente, quizá pueda salvarte." Esa fue la frase que utilizo el doctor Cowley para describir "La Hora Dorada." El nunca estaba satisfecho, "Hemos hecho un gran trabajo," dijo durante un recorrido por el nuevo centro de trauma, "pero no hemos hecho lo suficiente, la gente sigue muriendo innecesariamente."

Esto lo dijo hace mas de 40 años. Tuve la fortuna de conocerlo y estas palabras dejaron en mí una huella inmensa. Hoy a pesar de todo el trabajo que han hecho los cirujanos en el mundo, incluidos nosotros, podemos decir que esto sigue sucediendo, "la gente sigue muriendo innecesariamente" a pesar que cada vez ha disminuido mas la brecha, esto sigue sucediendo. ¿Pero cómo logramos disminuir esto al máximo?

Albert Einstein decía, "Locura es hacer lo mismo una y otra vez esperando obtener resultados diferentes," pero eso es lo que hacemos siempre, casi siempre hacemos lo mismo, es lo razonable, es como nos ha ido bien, es lo que esta proyectado, lo que dice la literatura, es la norma, el dogma. Atreverse a hacer algo diferente nos cuesta mucho trabajo, es salir del estado de confort, cambiar los paradigmas e ir en contra del establecimiento, esto nos puede salir muy caro, la crítica, la oposición, las posibles demandas, las auditorias y como dicen algunos amigos, éste con sus locuras y atrevimientos.

Nos enseñan a vivir con temor, y esto hace que las cosas no cambien o que lo hagan muy lentamente. Hay que atreverse a hacer cosas diferentes y esperar resultados diferentes, hay que soñar para lograr resultados imposibles. Es necesario luchar, perseverar, arriesgar, no tener miedo al fracaso, ser resiliente, adaptarse positivamente a las situaciones adversas. Tener creatividad e innovación, esto es lo que distingue a los lideres de los seguidores.

Se deben tener convicciones firmes y claras, porque si llega a tus manos un paciente que sufre un trauma o un accidente muy grave y te llega vivo, ¡tiene que salir vivo! La vida de esa persona esta en tus manos, tienes que tener el conocimiento, las destrezas y la seguridad para que los dos salgan adelante.

Cali lleva 4 décadas viviendo una época de violencia impresionante, en los 90 era una de las ciudades mas violentas del mundo, con una tasa de homicidios de más de 125 por 100. 000 habitantes; para que ustedes vean la magnitud de este número tasas por encima de 20 son consideradas una epidemia. Hoy hemos mejorado mucho pero aun es muy alta, la tasa de homicidios esta ligeramente por debajo de 50 por 100.000 .

iiPero esta desgracia la hemos convertido en una gracia!! Porque entre las dificultades se esconde la oportunidad. Aprendimos de esto, nos volvimos un equipo de médicos y cirujanos muy preparados para afrontar esta situación, se conoce que por cada muerto hay 4 a 6 heridos y para poder traer de nuevo a la vida a estos heridos hay que estar muy bien entrenados y consientes de los hechos.

Cuando iniciamos a finales de los años 80 se moría entre el 70 al $80 \%$ de los pacientes graves heridos por arma de fuego, hoy se muere el $20 \%$ de estos pacientes en este hospital.

La educación es el arma más poderosa que se puede usar para cambiar el mundo, dijo Nelson Mandela, la aplicamos nosotros en la cirugía de trauma, emergencias y cuidado intensivo. La habilidad del cirujano está en sus manos y en su cerebro, que le da seguridad, anticipación, y resolución. Tienes que hacer en la vida algo que te apasione, y nosotros tenemos esa pasión.

Hemos aprendido que no solo debemos comprometernos con el paciente que tenemos enfrente y que somos capaces de sacarlo adelante, por eso esta experiencia adquirida la estamos trasformando en ciencia, escribimos científicamente lo que hacemos para que los médicos y cirujanos, del otro lado del mundo lo lean y sí a nosostros nos va bien con las nuevas técnicas, con la innovación, con los nuevos cambios que nos atrevemos a hacer, es posible que a ellos tambien les vaya bien, entonces estamos contribuyendo con la educación y la investigación y lo que es mas valioso es que estamos ayudando con un grano de arena a salvar vidas acá y al otro lado del mundo, tenemos que ser cirujanos que dejamos huella y no solo cicatrices. 
Un Lider debe formar líderes, en el caso nuestro, se tiene un fin y un objetivo común, pero ellos, la nueva generación debe seguir innovando y debe seguir rompiendo paradigmas hasta que logremos reducir la brecha de la mortalidad al máximo. Hemos creado una liga, una familia, una hermandad, que se expande en Colombia y latinoamerica como los cirujanos pioneros que manejan el cuidado agudo del paciente, desde que ingresa a urgencias, se opera, se estabiliza, reanima en la unidad de cuidado intensivo y se rehabilita, estamos contribuyendo a disminuir los años de vida perdida, la morbilidad y la mortalidad.

¿Cómo se construye una verdadera carrera académica? Desde mi percepción, se debe disfrutar trabajando, tener pasión por lo que se hace, sentir placer, disfrutar la cirugía, y el trabajo en la unidad de cuidado intensivo, no tener estrés, o disminuirlo al maximo, desarrollar habilidades manuales y cerebrales, siempre ir con una, dos o tres jugadas adelante, aprender a manejar el equipo, que todos trabajen coordinados y por un fin comun, hay que ser un verdadero jugador de ajedrez.

Esa experiencia y ese conocimiento deben estar al alcance de todos y se debe transformar en un escrito; ese conocimiento debe convertirse en ciencia y debe ser transmitido. Ese legado por pequeño que sea cobra sentido cuando es útil para los demás.

El Dr Alonso Gomez, profesor emérito de cuidado intensivo un día me dijo que lo más importante de todo, es que seas capaz de formar una escuela, eso es lo que estamos construyendo, la escuela para Colombia y el mundo, de cirujanos de trauma y emergencias con una base solida en cuidado intensivo, cirujanos para la vida, comprometidos con sus pacientes, con la institución, con el país, y el conocimiento (Figs 1 and 2).

Hoy despues de las guerras de Irak y Afganistan los militares han establecido los 10-15 minutos de platino, hay que lograr el control en ese tiempo, una hora en un paciente grave es demasiado tiempo. Los médicos en el mundo estamos trabajando arduamente para lograr este objetivo. No esperemos que el mundo nos lo muestre, porque esto va a suceder, mostremosle nosotros al mundo que tenemos la capacidad, el valor, y lo más importante, los pacientes en riesgo, esta sera nuestra contribución para disminuir la brecha al maximo. Entre todos con el respaldo de la institución lo lograremos.

Albert Einstein decía, "La vida es muy peligrosa. No por las personas que hacen el mal, sino por las que se sientan a ver lo que pasa." Bienvenidos al futuro. Muchas gracias.

Carlos Alberto Ordoñez D MD, FACS

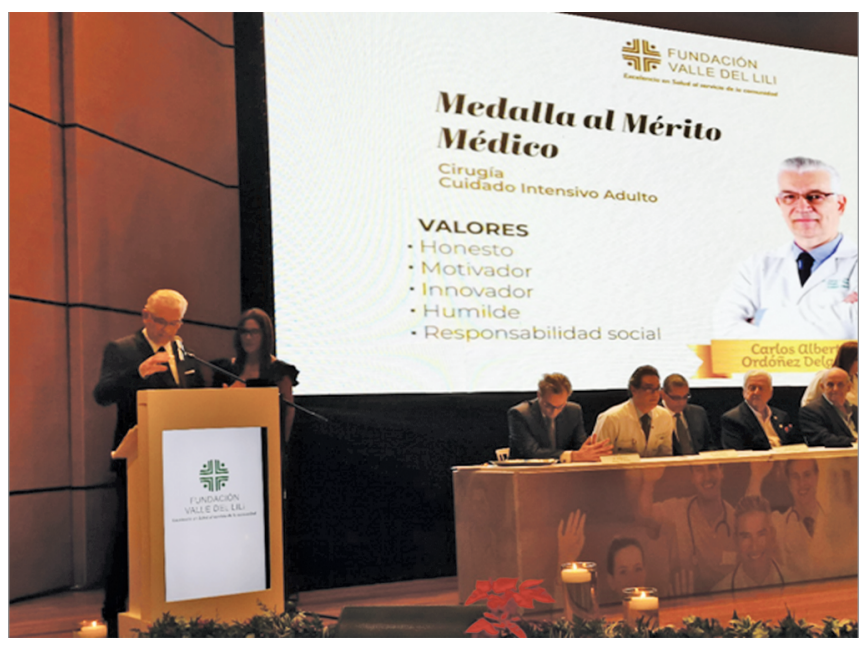

Fig. 1: Entrega de la Medalla al Mérito Médico con reconocimiento de los valores de honestidad, motivación, innovación, humildad y responsabilidad social. A la izquierda, el Dr Carlos A Ordoñez

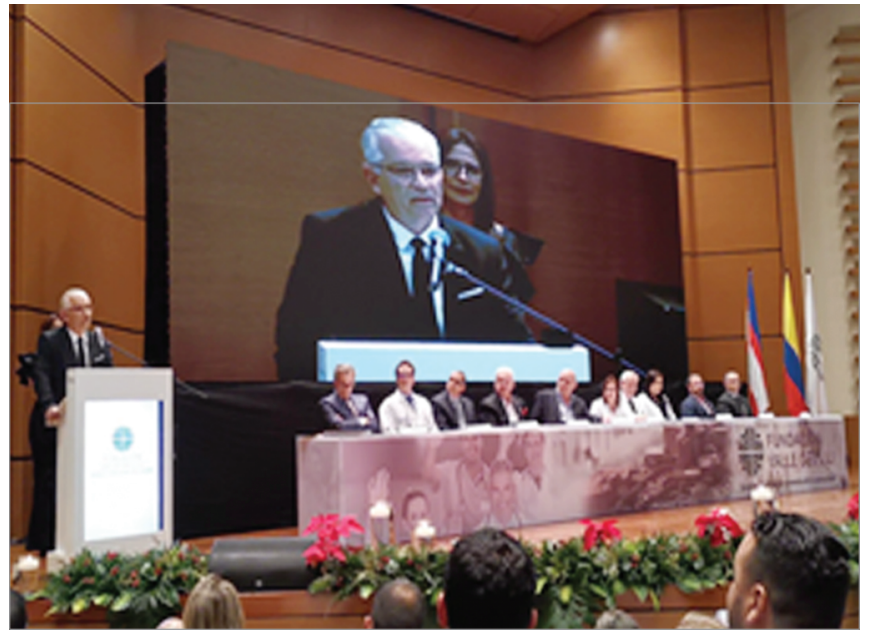

Fig. 2: Discurso del Dr Carlos A Ordoñez durante la ceremonia de entrega de la Medalla al Mérito. En la mesa de panelistas, se encuentra el personal directivo de la clínica y el señor alcalde Maurice Armitage 